\title{
OPEN The Wnt/ $\beta$-catenin signaling pathway has a healing ability for periapical periodontitis
}

\author{
Haruna Naruse ${ }^{1}$, Shousaku Itoh ${ }^{1}$, Yuki Itoh ${ }^{1}$, Takumi Kagioka ${ }^{1}$, Makoto Abe ${ }^{2}$ \& \\ Mikako Hayashi ${ }^{1}$
}

Various disease-related genes have recently been identified using single nucleotide polymorphisms (SNPs). This study identified disease-related genes by analyzing SNP using genomic DNA isolated from Japanese patients with periapical periodontitis. Results showed that the SNP in LRP5 demonstrated a significant genotypic association with periapical lesions (Fisher's exact test, $P<0.05$ ). We constructed an in vivo murine periapical periodontitis model to confirm the Wnt/ $\beta$ catenin signaling pathway's role in developing and healing periapical periodontitis. We observed that administration of the Wnt/ $\beta$-catenin signaling pathway inhibitor enlarged the periapical lesion. Moreover, applying lithium chloride ( $\mathrm{LiCl}$ ) to root canals accelerated periapical periodontitis healing. Histological analysis demonstrated that the expression levels of Col1a1 and Runx2 increased in the $\mathrm{LiCl}$ application group compared to that in the control group. Furthermore, many CD45R-positive cells appeared in the periapical lesions in the $\mathrm{LiCl}$ application group. These results indicated that $\mathrm{LiCl}$ promoted the healing of periapical periodontitis by inducing bone formation and immune responses. Our findings suggest that the $W n t / \beta$-catenin signaling pathway regulates the development of periapical periodontitis. We propose a bioactive next-generation root canal treatment agent for this dental lesion.

Periapical periodontitis is an inflammatory disease of periapical tissues caused by an infection in the root canal system due to inadequate root canal treatment. The invasion of microbes from the root canal system into the periapical tissues induces host immune responses ${ }^{1,2}$. During the host defense reaction, the host's cells produce various inflammatory and anti-inflammatory molecules, and some inflammatory molecules induce osteoclast differentiation ${ }^{3}$. Then, bone homeostasis is disrupted in case of periapical periodontitis, which promotes increased rates of bone resorption, resulting in the formation of periapical lesions that are observed as radiolucent areas ${ }^{3}$. Several disease-related genes have been identified for various multifactorial diseases using single nucleotide polymorphisms (SNPs) in recent years. In case-control studies, some researchers reported that $I L-1 \beta$ polymorphism is associated with periapical periodontitis ${ }^{4-6}$. Meanwhile, although several previous SNP studies reported inflammatory cytokines related to periapical periodontitis, only a few reports described other factors ${ }^{7,8}$. In the field of bone metabolism, studies reported that the SNP located in exon 18 of the low-density lipoprotein receptor-related protein 5 (LRP5, Wnt coreceptor) gene, caused due to an amino acid change (3989C>T, A1330V), is associated with osteoporosis among patients in Japan, Europe, America, and South America ${ }^{9-18}$. Therefore, in this study, we focused on the SNP of LRP5 related to bone metabolism.

The Wnt $/ \beta$-catenin signaling pathway plays a significant role in the maintenance of bone homeostasis ${ }^{19,20}$. Wnt proteins bind to their receptor Frizzled and the coreceptor LRP 5/6, suppressing the phosphorylation of $\beta$-catenin using glycogen synthase kinase- $3 \beta$ (GSK-3 $\beta$ ). The stabilized $\beta$-catenin accumulates in the cytoplasm. Accumulation and nuclear translocation of $\beta$-catenin enable the association with the transcription factor LEF-1/TCF and activate various target genes (canonical pathway). It has been reported that $\mathrm{Li}^{+}$can activate the Wnt/ $\beta$-catenin signaling pathway by inhibiting GSK-3 $\beta$ activity ${ }^{21}$. Experimental studies also reported that lithium chloride $(\mathrm{LiCl})$ induces hard tissue formation in vivo and in vitro ${ }^{21,22}$. Conversely, there are also the report describing that the Wnt/ $\beta$-catenin signaling pathway regulates the differentiation of $\mathrm{T}$ cells and $\mathrm{B}$ cells ${ }^{23}$. For example, in one study, the inhibition of the Wnt canonical pathway blocked the transition from double-negative $\mathrm{T}$ cells to double-positive $\mathrm{T}_{\text {cells }}{ }^{24}$. In another study, LEF-1-deficient mice exhibited defects in pro-B-cell proliferation and survival both in vitro and in vitro ${ }^{23}$. Periapical periodontitis is caused by the host's defense against microbial

\footnotetext{
${ }^{1}$ Department of Restorative Dentistry and Endodontology, Osaka University Graduate School of Dentistry, 1-8 Yamadaoka Suita, Osaka 565-0871, Japan. ${ }^{2}$ Department of Oral Anatomy and Developmental Biology, Osaka University Graduate School of Dentistry, Osaka, Japan. ${ }^{\circledR}$ email: ito@dent.osaka-u.ac.jp
} 


\begin{tabular}{|l|l|l|l|}
\hline \multirow{2}{*}{ LRP5 (rs3736228) } & \multicolumn{3}{|l|}{ Genotype frequency } \\
\cline { 2 - 4 } & Case & Control & P value \\
\hline C/C & 0.36 & 0.54 & NS \\
\hline C/T & 0.62 & 0.46 & $0.00396^{*}$ \\
\hline T/T & 0.02 & 0.00 & NS \\
\hline
\end{tabular}

Table 1. Results of case-control comparisons for the association of SNPs in LRP5. A statistically significant difference occurred in $\mathrm{C} / \mathrm{T}$ frequency (case group vs. control group, $P<0.05$ as assessed by Fisher's exact test).

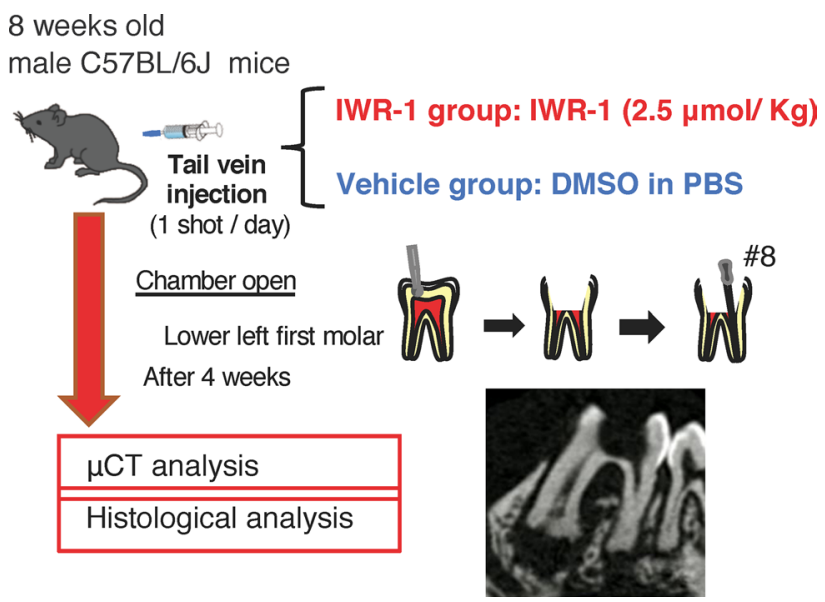

Figure 1. In vivo inhibition model of the Wnt/ $\beta$-catenin signaling pathway. The pulp chamber of the lower first molar was exposed to the oral cavity. IWR-1 or DMSO in PBS was administered into the tail vein once a day from the day of pulp exposure. At 4 weeks after the first administration, the periapical lesions were evaluated by micro-CT and histological analysis. A representative image for the micro-CT is shown.

invasion and alveolar bone resorption by disrupting bone homeostasis. Hence, we focused on the role of the Wnt/ $\beta$-catenin signaling pathway in both immunity and bone homeostasis.

We hypothesized that the A1330V variant of LRP5 might be associated with apical periodontitis. In the present study, we selected individuals with periapical lesions measuring $>3 \mathrm{~mm}$ in diameter as the case group. We performed a genetic polymorphism analysis for these individuals and a control group (individuals with no apical lesion). We found that the A1330V variant of LRP5 was associated with periapical periodontitis. To confirm the $\mathrm{Wnt} / \beta$-catenin pathway's role in periapical periodontitis development, we practiced an in vivo murine periapical periodontitis model. Although the administration of the Wnt/ $\beta$-catenin pathway inhibitor enlarged the apical lesion, applying $\mathrm{LiCl}$ into the root canals accelerated periapical periodontitis healing. Our findings demonstrated that the Wnt/ $\beta$-catenin signaling pathway regulates periapical periodontitis development. We propose a novel therapeutic strategy for this dental lesion.

\section{Results}

LRP5 SNP is related to periapical periodontitis. The mean age of patients was $48.2 \pm 20.3$ years in the case group and 59.8 \pm 17.4 years in the control group. The case group consisted of 19 males and 31 females, and the control group consisted of 12 males and 18 females. The mean diameter of the periapical lesion was $6.0 \pm 2.7 \mathrm{~mm}$. Table 1 shows the results of genotyping of LRP5 SNP (A1330V) for the case and control groups. The frequency of $\mathrm{C} / \mathrm{T}$ was statistically significant between the groups (case group vs. control group, $P=0.00396$ ).

Inhibition of the Wnt/ $\beta$-catenin signaling pathway spreads the periapical lesion. The lower left first molar pulp was exposed, and the inhibitor of the Wnt/ $\beta$-catenin signaling pathway (IWR-1) ${ }^{25}$ was administered once a day after the exposure (Fig. 1). The periapical lesion volume was analyzed by micro-CT, showing that the periapical lesion volume in the IWR-1 group was significantly larger than that in the vehicle group at 4 weeks (vehicle group: $1.46 \pm 0.01 \mathrm{~mm}^{3}$, IWR-1 group: $1.65 \pm 0.33 \mathrm{~mm}^{3}$ ) (Fig. 2A). To confirm the suppression of the Wnt $/ \beta$-catenin signaling pathway, we evaluated the expression of Axin 2 by in situ hybridization. The expression of Axin2 was decreased in the IWR-1 group compared to that in the vehicle group after administration of IWR-1 (Fig. 2B). H-E staining results revealed the presence of heavy hematoxylin-concentrated cells around the root and the alveolar bone (Fig. 3). We observed the expression of Runx2 and Colla1 on the surface of the alveolar bone around the periapical lesion in both groups, which was decreased in the IWR-1 group compared to that in the vehicle group (Fig. 3, Supplementary Fig. S1). 
A

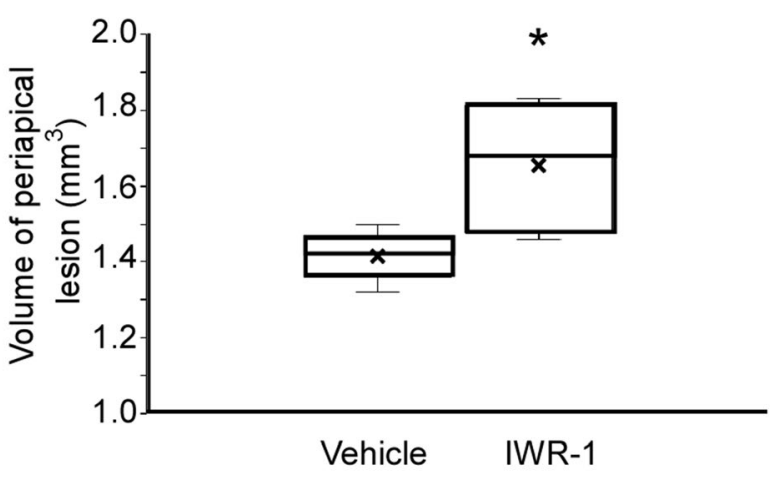

B

Vehicle

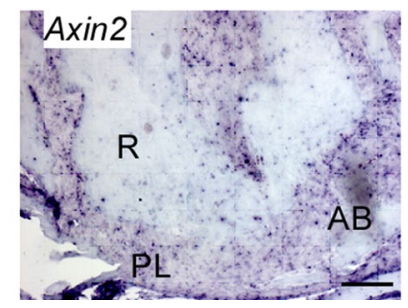

IWR-1

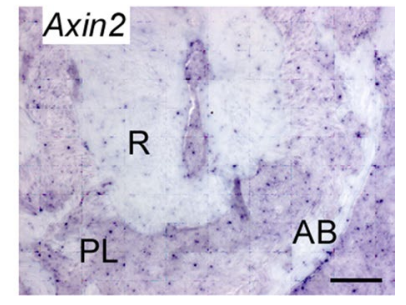

Figure 2. IWR-1 injection inhibited the Wnt/ $\beta$-catenin signaling pathway and spread the periapical lesion. (A) The quantification of periapical lesion volumes of the vehicle group (white bar, mean $\pm \mathrm{SD}, \mathrm{n}=4$ ) and the IWR-1 treatment group (black bar, mean $\pm S D, n=4$ ). Two-tailed paired Student's $t$ test, ${ }^{\star} P<0.05$. (B) In situ hybridization for Axin 2 in the periapical lesion at 4 weeks after chamber opening. $R$ root, $P L$ periapical lesion, $A B$ alveolar bone. Scale bars $=100 \mu \mathrm{m}$.

Activation of the $\mathrm{Wnt} / \beta$-catenin signaling pathway reduced the periapical lesion volume. $\mathrm{LiCl}$ is a chemical known to activate the Wnt/ $\beta$-catenin signaling pathway ${ }^{21}$. Hence, we applied it to the root canal for 4 weeks after exposure, followed by histological analysis and micro-CT analysis (Fig. 4). The periapical lesion volume in the $\mathrm{LiCl}$ application group was significantly smaller than that in the control group at 4 weeks after root canal treatment (control group: $3.44 \pm 0.30 \mathrm{~mm}^{3}, \mathrm{LiCl}$ application group: $2.32 \pm 0.30 \mathrm{~mm}^{3}$ ) (Fig. 5A). To confirm the activation of the Wnt/ $\beta$-catenin signaling pathway, we evaluated the expression of Axin 2 by in situ hybridization. We observed that the $\mathrm{LiCl}$ application group showed a higher expression level of Axin 2 than that in the control group (Fig. 5B). The results of H-E staining of fibroblasts and angiogenesis appeared in both groups (Fig. 6). In the control group, the expression areas of Runx2 and Colla1 were observed only in the bone surface surrounding the periapical lesion (Fig. 6). Conversely, in the $\mathrm{LiCl}$ application group, the expression areas of Runx2 and Colla1 were observed not only in the bone surface surrounding the periapical lesion but also in the periapical lesion (Fig. 6). There was no difference in CD3-positive T cells between the two groups (Fig. 7). In contrast, many CD45R-positive $\mathrm{B}$ cells were observed in the $\mathrm{LiCl}$ application group compared to that in the control group (Fig. 7).

\section{Discussion}

In clinical cases, some patients have no radiolucent area in the periapical lesion despite the apparent occurrence of bacterial infection in the root canal. Conversely, there are also some patients who show a radiolucent area in the periapical lesion despite tight root canal filling. Based on these various clinical cases, we speculated that some factors other than bacterial infection are involved in the onset of periapical periodontitis. Periapical periodontitis is caused by the immune response to foreign factors, such as bacterial invasions, resulting in the subsequent absorption of the alveolar bone by osteoclasts, induced by the production of inflammatory cytokines during the immune response. Thus, periapical periodontitis is caused by bacterial infection factors and host factors such as immune responses and bone metabolism. Recently, focusing on this host fact, several disease-related genes have been identified using SNPs for various multifactorial diseases. Therefore, we decided to analyze the genes related to periapical periodontitis. We chose to extract a candidate gene related to immune response and bone metabolism, i.e., LRP5, a gene involved in the bone metabolism system ${ }^{9-18}$. We found that the distribution of LRP5 SNP (rs3736228) was significantly biased in hetero types (Table 1). Researchers reported that A1330V is associated with $\mathrm{T}$ cell factor/lymphoid enhancer factor family (Tcf/Lef) activity in an in vitro study ${ }^{18}$. Therefore, in this study, we successfully identified a new SNP associated with the onset of periapical periodontitis.

To address the mechanism and factors related to periapical periodontitis, we used a recently-developed murine periapical periodontitis mode ${ }^{26,27}$. Recently, some factors related to periapical periodontitis have been 


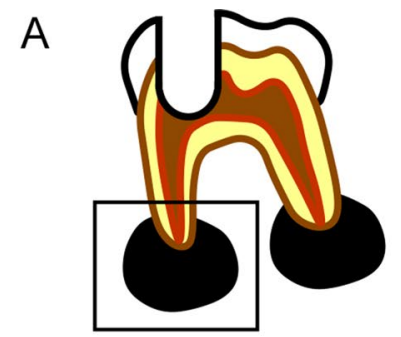

B

Vehicle
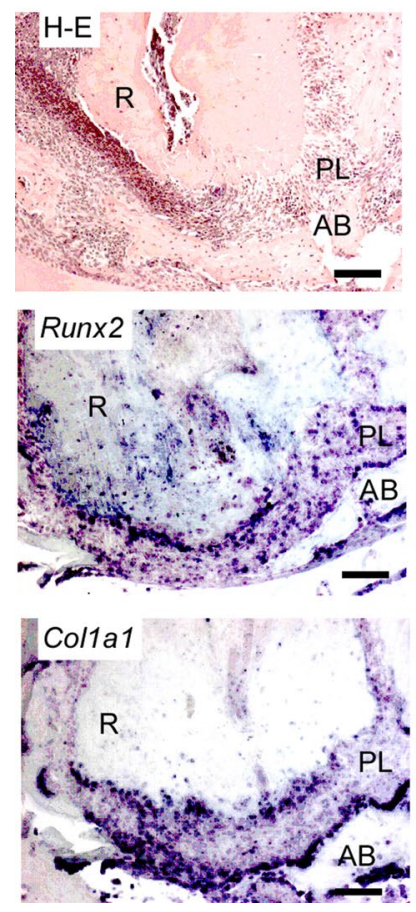

IWR-1
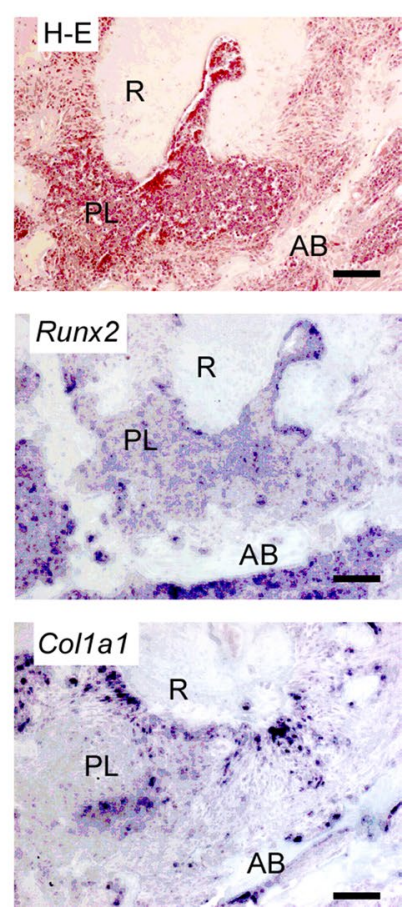

Figure 3. Inhibition of the Wnt/ $\beta$-catenin signaling pathway resulted in suppressed expression levels of osteoblast differentiation markers. Periapical lesion tissues within a rectangular frame (A) of the vehicle group and the IWR-1 group were subjected to H-E staining and in situ hybridization for Runx 2 and Col1a1 (B). $R$ root, $P L$ periapical lesion, $A B$ alveolar bone. Scale bars $=100 \mu \mathrm{m}$.

clarified $^{26,27}$. From the result of our SNP analysis, we try to clarify the role of the Wnt $/ \beta$-catenin signaling pathway for periapical periodontitis. First, we analyzed the volume of the periapical lesion when the Wnt $/ \beta$-catenin signaling pathway was suppressed. IWR-1, a low-molecular-weight compound, can penetrate the cell membrane easily and effectively inhibits cellular signaling pathways ${ }^{25}$. IWR-1 stabilizes the complex of Axin2, APC, Ck1, and Gsk $3 \beta$ and then promotes the degradation of $\beta$-catenin. In the present study, an enlarged volume of the periapical lesion was observed in the IWR-1 group compared to that in the vehicle group (Fig. 2A). Therefore, we performed in situ hybridization to verify whether the enlarged periapical lesion volume in the IWR-1 group was caused by the inhibition of the Wnt/ $\beta$-catenin signaling pathway by IWR-1. We found that Axin 2 expression was suppressed in the IWR-1 group (Fig. 2B). Furthermore, the expression levels of Colla1 and Runx2, osteoblast differentiation markers, were decreased in the IWR-1 group (Fig. 3, Supplementary Fig. S1). There was no change in osteoclast differentiation (Supplementary Fig. S2). These results demonstrated that the inhibition of the Wnt/ $\beta$-catenin signaling pathway by the administration of IWR-1 suppressed the differentiation of osteoblasts and then promoted the enlarged volume of the periapical lesion.

The inhibition of the Wnt/ $\beta$-catenin signaling pathway resulted in the enlargement of the periapical lesion. According to the IWR-1 experiment results, we speculated that the activation of the Wnt/ $\beta$-catenin signaling pathway reduced the volume of the periapical lesion volume. We next explored the relationship between the activation of the Wnt/ $\beta$-catenin signaling pathway and the volume of the periapical lesion. It is known that $\mathrm{LiCl}$ activates the Wnt canonical pathway. $\mathrm{LiCl}$ also inhibits the activity of GSK3 $\beta$, induces the accumulation of $\beta$-catenin in the cytoplasm, and activates transcription by promoting the intranuclear translocation of $\beta$-catenin. Therefore, we examined whether $\mathrm{LiCl}$ exhibits a healing ability for periapical periodontitis using a murine periapical periodontitis model ${ }^{26,27}$. The periapical lesion volume in the $\mathrm{LiCl}$ application group was significantly reduced after 4 weeks of $\mathrm{LiCl}$ application (Fig. 5A). Next, we performed in situ hybridization to verify whether 
8 weeks old male C57BL/6J mice

Chamber open

Lower left first molar

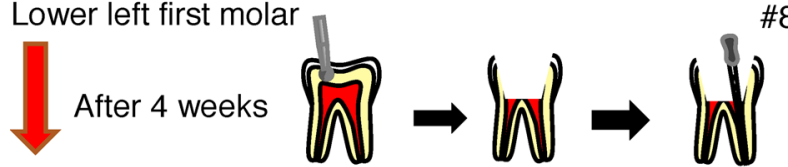

$\underline{\text { LiCl Application }}$

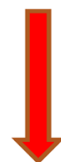

After 4 weeks

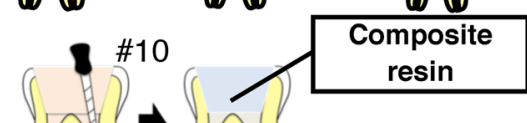

\begin{tabular}{|l|}
\hline$\mu C T$ analysis \\
\hline \hline Histological analysis \\
\hline
\end{tabular}

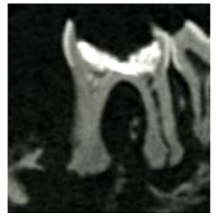

Figure 4. In vivo activation model of the Wnt $/ \beta$-catenin signaling pathway. The pulp chamber of the lower first molar was exposed to the oral cavity. At 4 weeks after pulp exposure, root canal cleaning was performed using the \# $10 \mathrm{~K}$ file. After root canal cleaning, $\mathrm{LiCl}$ was applied to the root canal, and the periapical lesions were evaluated by micro-CT and histological analysis. A representative image for the micro-CT is shown.

\section{A}

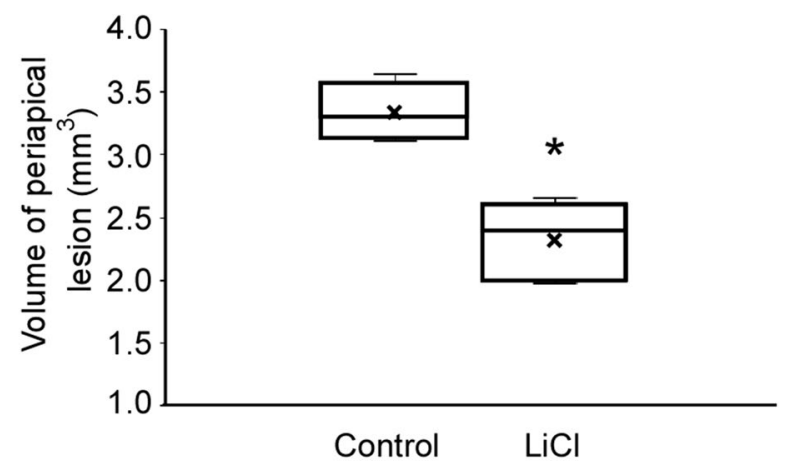

B
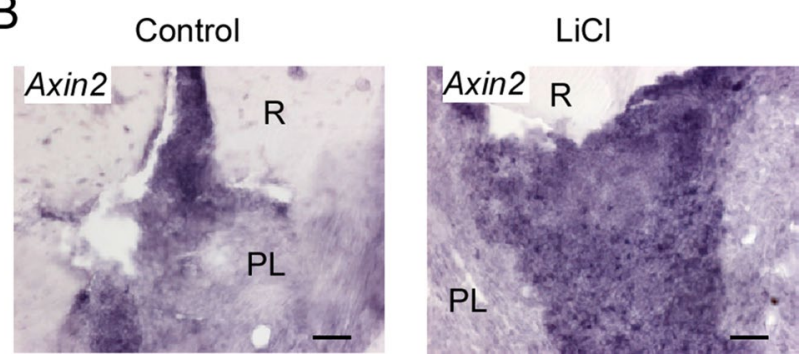

Figure 5. Activation of the Wnt $/ \beta$-catenin signaling pathway reduced the periapical lesion volume. (A) Quantification of periapical lesion volumes of the control group (white bar, mean $\pm \mathrm{SD}, \mathrm{n}=4$ ) and the $\mathrm{LiCl}$ application group (black bar, mean $\pm \mathrm{SD}, \mathrm{n}=4$ ). Two-tailed paired Student's $t$ test, ${ }^{\star} P<0.05$. (B) In situ hybridization for Axin 2 in the periapical lesion at 4 weeks after chamber opening. $R$ root, PL periapical lesion. Scale bars $=50 \mu \mathrm{m}$.

the reduced periapical lesion volume observed in the $\mathrm{LiCl}$ application group was caused by the activation of the $\mathrm{Wnt} / \beta$-catenin signaling pathway by LiCl. Our results showed that the tissue sections at $24 \mathrm{~h}$ after LiCl application demonstrated high expression of Axin2 (Fig. 5B). This result suggested that $\mathrm{LiCl}$ activates the Wnt/ $\beta$-catenin signaling pathway in the periapical lesion. In addition, $\mathrm{LiCl}$ application resulted in a higher expression of Col1a 1 and Runx2 than that in the control group (Fig. 6, Supplementary Fig. S1). These results demonstrated that the 


\section{Control}
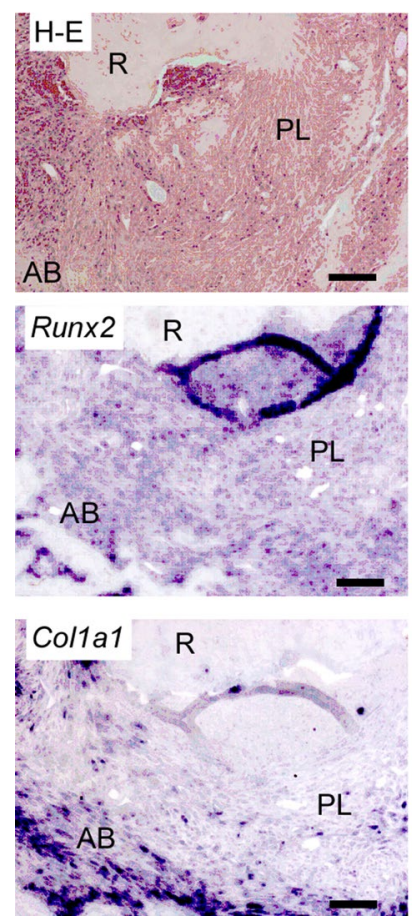

$\mathrm{LiCl}$
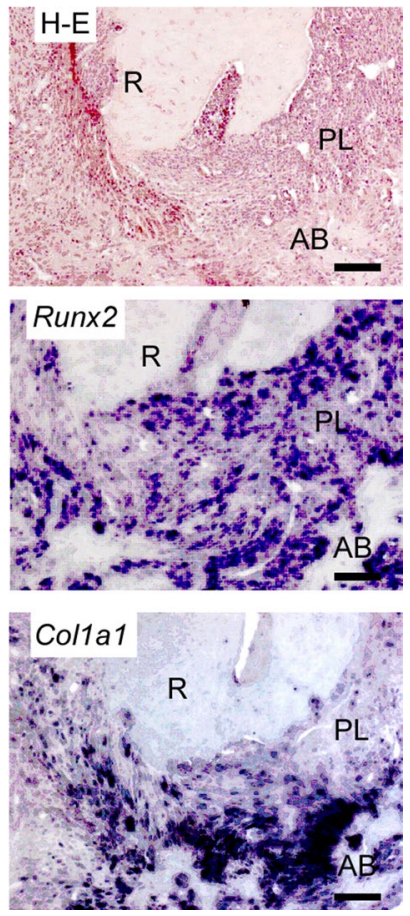

Figure 6. Activation of the Wnt/ $\beta$-catenin signaling pathway resulted in the spreading of the expression areas of osteoblast differentiation markers. Periapical lesion tissues of the control group and the $\mathrm{LiCl}$ application group were subjected to $\mathrm{H}-\mathrm{E}$ staining and in situ hybridization for Runx2 and Colla1. $R$ root, PL periapical lesion, $A B$ alveolar bone. Scale bars $=100 \mu \mathrm{m}$.
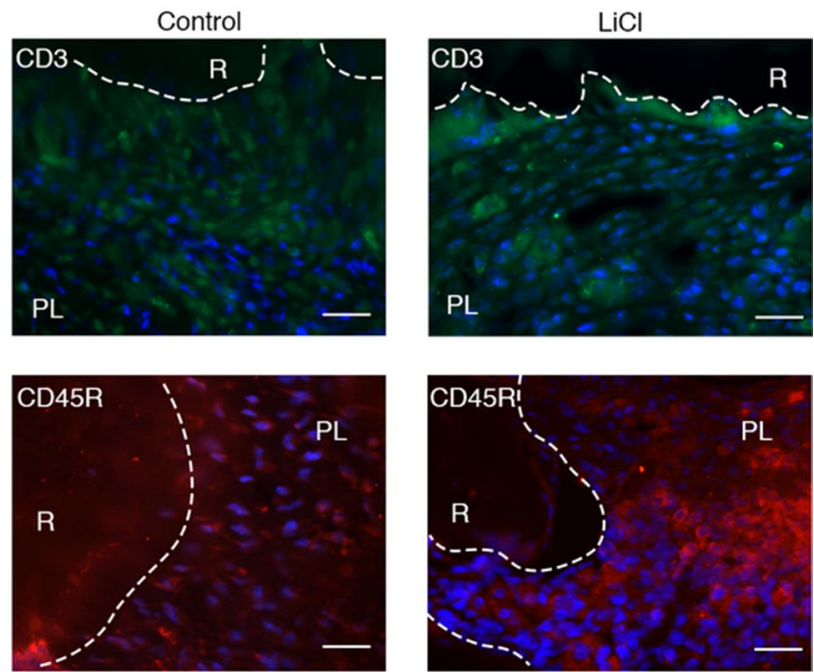

Figure 7. A large number of CD45R-positive cells were observed in the $\mathrm{LiCl}$ application group. Periapical lesion tissues of the control group and the $\mathrm{LiCl}$ application group were visualized by staining with Alexa 488 (CD3), Alexa 594 (CD45R), and DAPI. $R$ root, $P L$ periapical lesion. Scale bars $=50 \mu \mathrm{m}$.

activation of the $\mathrm{Wnt} / \beta$-catenin signaling pathway by $\mathrm{LiCl}$ induces the differentiation of osteoblasts and promotes the healing of periapical periodontitis.

Because the Wnt canonical pathway regulates the differentiation of immune cells, we analyzed the effect of $\mathrm{LiCl}$ application on the differentiation of immune cells. Although no difference in the number of CD3-positive cells between the $\mathrm{LiCl}$ application group and the control group (Fig. 7) existed, the number of CD45R-positive cells in the $\mathrm{LiCl}$ application group was higher (Fig. 7). CD45R is expressed from pro-B cells to mature B cells, and a previous study showed that the $\mathrm{Wnt} / \beta$-catenin signaling pathway positively regulates the proliferation ability 
of $\mathrm{B}$ cells ${ }^{23}$. This is consistent with the result of the increase in the number of B cells in the periapical lesion of the $\mathrm{LiCl}$ application group. Although $\mathrm{LiCl}$ reportedly maintains $\mathrm{T}$ cells in an undifferentiated state ${ }^{24}$, no change occurred in CD3-positive cells. This discrepancy might be caused due to the different application methods, i.e., the local application into the root canal in this study and the systemic administration in the previous study ${ }^{24}$.

In summary, $\mathrm{LiCl}$ promotes the healing of periapical periodontitis by inducing bone formation and immune responses. Although conventional root canal treatment agents such as calcium hydroxide primarily target the disinfection of bacteria in the root canal, $\mathrm{LiCl}$ targets the $\mathrm{Wnt} / \beta$-catenin signaling pathway by regulating bone metabolism and immune response. Therefore, our results suggest that $\mathrm{LiCl}$ could be used as a bioactive nextgeneration root canal treatment agent.

\section{Methods}

Sample population and genotyping. This study was approved by the research Ethics Review Committee of Osaka University (\#450), Osaka, Japan and the Ethics Review Committee of Osaka University Dental Hospital (\#H25-E22). All participants signed an informed consent and provided a sample as a source of genomic DNA. Participants whose radiographic records showed periapical lesions measuring $>3 \mathrm{~mm}$ in diameter were assigned to the case group $(n=50)$. Participants who presented with no periapical lesions after root canal treatment were assigned to the control group $(n=30)$. Buccal mucosa was collected by wiping with a sterile swab. The tip of the swab was immersed in a lysis buffer [100 mM Tris- $\mathrm{HCl}$ (pH 8.5), 5 mM EDTA, 0.2\% SDS, $200 \mathrm{mM} \mathrm{NaCl}, 100 \mu \mathrm{g}$ proteinase $\mathrm{K} / \mathrm{ml}$ ] and reacted at $55^{\circ} \mathrm{C}$ for $24 \mathrm{~h}$. After the reaction, genomic DNA was prepared by isopropanol precipitation and ethanol precipitation. Genomic DNA was diluted in $100 \mu \mathrm{l}$ TE solution and stored at $-80{ }^{\circ} \mathrm{C}$. PCR was performed for LRP5 (dbSNP ID: rs3736228) gene amplification using TaqMan GTXpressTM Master Mix and TaqMan SNP Genotyping Assays (Applied Biosystems, California, USA). All methods were performed following the relevant guidelines and regulations set forth by the Declaration of Helsinki. Informed written consent was obtained from all participants.

Murine periapical periodontitis model. The study was approved by the research Ethics Committee of the Osaka University, Osaka, Japan, and all experiments were performed according to the guidelines related to animal care (AD-26-011-0) and compliant with ARRIVE guidelines (http://www.nc3rs.org.uk/page.asp?id= 1357). C57BL/6 J mice (8-week-old) were intraperitoneally anesthetized with Domitor $(0.3 \mathrm{mg} / \mathrm{kg}$ ) (Nippon Zenyaku Kogyo Co., Fukushima, Japan), Dolmicam (4 mg/kg) (Astellas Pharma Inc., Tokyo, Japan), and Betferar (5 mg/kg) (Meiji Seika Pharma, Tokyo, Japan). The pulp chamber of the lower first molar was accessed with a \# 1/4 round bar (Dentsply, Ballaigues, Switzerland) equipped with an electric engine (VIVA MATE G 5, NSK, Tochigi, Japan). The root canals were instrumented with the \# $08 \mathrm{~K}$ file (Dentsply) under the operating microscope and left exposed to the oral cavity. Mice were monitored daily for clinical signs of abnormal posture, lack of grooming, weight loss exceeding $20 \%$ of body weight, and anorexia. Though the presence of any of these findings was considered as an endpoint and mice would be euthanized using a $\mathrm{CO}_{2}$ chamber, we did not observe any of these findings in the mice used for experiments.

IWR-1 administration. The solutions $(0.04 \mathrm{ml})$ adjusted as follows were administered into the tail vein once a day from the day of pulp exposure (Fig. 1). The solutions were adjusted as follows: control solution: DMSO (Wako Pure Chemical Industries, Osaka, Japan) was diluted in phosphate-buffered saline (PBS) (Final concentration: 5\%). IWR-1 (Sigma-Aldrich, Missouri, USA) solution (IWR-1: $2.5 \mu \mathrm{mol} / \mathrm{kg}$ ) was diluted in the control solution. At 4 weeks after the first administration, the periapical lesion volume was measured (each group, $\mathrm{n}=4$ ).

$\mathrm{LiCl}$ application. At 4 weeks after pulp exposure, root canal cleaning was performed using a \# $10 \mathrm{~K}$ file (Dentsply). $\mathrm{LiCl}$ was ground into the small size powder within $100 \mu \mathrm{m}$. After root canal cleaning, $\mathrm{LiCl}$ (Wako Pure Chemical Industries, Ltd.) was applied into the root canal ( $0.025 \mathrm{~g}$ per each root canal as a powder) with the slightly moistened \# $10 \mathrm{~K}$ file (Dentsply) under the operating microscope. Root canals of the control group did not contain anything. The pulp chamber was closed with the bonding material Clear fill bond SE ONE (Kuraray Noritake Dental, Tokyo, Japan), and a composite resin (MI flow: GC, Tokyo, Japan).

Micro-CT measurement. The volume of the periapical lesion was measured by micro-computed tomography (CT) (R_mCT 2: Science Mechatronics, Tokyo, Japan) performed on the lower first molar. The photographing conditions were set as follows: tube voltage $90 \mathrm{kV}$, tube current $160 \mu \mathrm{A}$, and slice width $5 \mu \mathrm{m}$. The obtained images were analyzed using SimpleViewer software (Science Mechatronics). Based on the method described by Kalatizis-Sousa et al. ${ }^{28}$ and, Yoneda et al. ${ }^{29}$, the periapical lesion volume was calculated using the bone morphometry software (TRI 3D-BON: RATOC, Osaka, Japan). The lesion volume was defined as the periapical transmission image volume and compared between experimental groups as previously reported ${ }^{27}$. The Student's $t$ test evaluated the statistically significant differences in the lesion volume $(\alpha=0.05)$.

Sample preparation for histological analysis. After subjecting the mice to the experiments described above, they were reflux-fixed in $4 \%$ paraformaldehyde (PFA) solution. The mandibles were collected, immersed, and fixed in 4\% PFA solution for $24 \mathrm{~h}$, followed by demineralization with $10 \%$ EDTA solution for 2 weeks. After demineralization, the samples were dehydrated with an ascending ethanol series and embedded in paraffin. Then, thin slices with a thickness of $9 \mu \mathrm{m}$ were prepared. 
Hematoxylin-eosin staining. Paraffin sections were deparaffinized, washed with water, and reacted with Mayer's hematoxylin solution (Muto, Osaka, Japan) for $7 \mathrm{~min}$. Then, the sections were washed with running water for $20 \mathrm{~min}$ and stained with eosin solution (Merck, Darmstadt, Germany) for 5 min, dehydrated with ethanol and decolorized, penetrated with xylene, and sealed with 50\% glycerol/PBS. The apical lesion and the surrounding bone were observed under an optical microscope (Axioskop 2 plus; Carl Zeiss, Aalen, Germany).

In situ hybridization. Paraffin sections were deparaffinized, washed with $0.01 \mathrm{M}$ PBS, fixed with $4 \%$ PFA for $10 \mathrm{~min}$, and washed again with PBS. The sections were reacted with $1 \mu \mathrm{g} / \mathrm{ml}$ protease K (Takara Bio, Shiga, Japan) for $5 \mathrm{~min}$ and then post-fixed with 4\% PFA. This was followed by acetylation with $0.1 \mathrm{M}$ triethanolamine containing $0.25 \%$ acetic anhydride and washing with $0.01 \mathrm{M} \mathrm{PBS}$. Prehybridization was carried out at $55^{\circ} \mathrm{C}$ for $1 \mathrm{~h}$, and hybridization was carried out overnight at $70^{\circ} \mathrm{C}$ using digoxigenin-labeled RNA probes, Axin2; addgene \#21277, NM_015732, nt1774-2787, Col1a1; M_007742, nt29553415, Runx2; AF010284, nt922_1746. After hybridization, the sections were placed in fivefold concentration sodium citrate solution (5-SSC) for $20 \mathrm{~min}$. They were reacted with 0.2 -SSC at $70{ }^{\circ} \mathrm{C}$ for $20 \mathrm{~min}$ and left to stand for $5 \mathrm{~min}$ in 0.2 -SSC in maleate buffer (MBA) for $5 \mathrm{~min}$. Then, blocking was performed for $2 \mathrm{~h}$ with a blocking solution containing $5 \%$ goat serum (Vector Laboratories, California, USA). After reacting with alkaline phosphatase (AP)-labeled anti-digoxigenin antibody (1:5000) (Roche, Basel, Switzerland) at $4{ }^{\circ} \mathrm{C}$ for $24 \mathrm{~h}$, the sections were washed with MBA supplemented with $0.1 \%$ Tween 20 , followed by washing with distilled water. The sections were reacted with BM Purple AP (Roche) as a substrate for $6 \mathrm{~h}$ at room temperature, after which they were washed with PBS and sealed with $50 \%$ glycerol/PBS. The apical lesion and the surrounding bone were observed under an optical microscope.

Immunohistochemistry. The paraffin sections were deparaffinized, washed with Tris-buffered saline (TBS), reacted at $100{ }^{\circ} \mathrm{C}$ for $10 \mathrm{~min}$ in a citrate buffer, and allowed to stand at room temperature for $30 \mathrm{~min}$. After blocking for $1 \mathrm{~h}$ with $10 \%$ goat serum-containing blocking solution (10\% goat serum/TBS), each antibody was reacted overnight at room temperature. The concentration of each antibody was 1:100 for CD3 (ab16669: Abcam, Cambridge, United Kingdom) and 1:50 for CD45R (ab64100: Abcam) for the experiments. After reaction with the primary antibody, the sections were washed with TBS and incubated with 1:500 Alexa 488-goat anti-rabbit IgG (Invitrogen, California, USA) or 1:500 Alexa 594-goat anti-rat IgG (Abcam) for $2 \mathrm{~h}$. Then, the sections were washed and reacted with DAPI (4',6-diamidino-2-phenylindole) (Sigma) for $15 \mathrm{~min}$. After the reaction, they were washed and sealed with $50 \%$ glycerol. The apical lesion and the surrounding bone were visible under a fluorescence microscope.

Statistical analyses. Results are presented as mean \pm SD. For SNP analyses, comparisons between groups were performed using Fisher's exact test $(\alpha=0.05)$. The Student's $t$-test quantified the differences in the periapical lesion volume.

Received: 13 May 2021; Accepted: 15 September 2021

Published online: 04 October 2021

\section{References}

1. Stashenko, P., Teles, R. \& D'Souza, R. Periapical inflammatory responses and their modulation. Cri. Rev. Oral Biol. Med. 9, 498-521 (1998).

2. Nair, P. N. Pathogenesis of apical periodontitis and the causes of endodontic failures. Crit. Rev. Oral Biol. Med. 15, 348-381 (2004).

3. Graves, D. T., Oates, T. \& Garlet, G. P. Review of osteoimmunology and the host response in endodontic and periodontal lesions. J. Oral Microbiol. 3, 5304 (2011).

4. Dill, A. et al. Analysis of multiple cytokine polymorphisms in individuals with untreated deep carious lesions reveals IL1B (rs1143643) as a susceptibility factor for periapical lesion development. J. Endod. 41, 197-200 (2015).

5. Iglesias-Linares, A. et al. Solano-Reina, Interleukin 1 gene cluster SNPs (rs1800587, rs1143634) influences post-orthodontic root resorption in endodontic and their contralateral vital control teeth differently. Int. Endod. J. 45, 1018-1026 (2012).

6. de Sa, A. R. et al. Association of CD14, IL1B, IL6, IL10 and TNFA functional gene polymorphisms with symptomatic dental abscesses. Int. Endod. J. 40, 563-572 (2007).

7. Menezes-Silva, R., Khaliq, S., Deeley, K., Letra, A. \& Vieira, A. R. Genetic susceptibility to periapical disease: Conditional contribution of MMP2 and MMP3 genes to the development of periapical lesions and healing response. J. Endod. 38, 604-607 (2012).

8. Maheshwari, K. et al. Heat shock 70 protein genes and genetic susceptibility to apical periodontitis. J. Endod. 42, 1467-1471 (2016).

9. Chubachi, S. et al. Polymorphism of LRP5 gene and emphysema severity are associated with osteoporosis in Japanese patients with or at risk for COPD. Respirology (Carlton, Vic.) 20, 286-295 (2015).

10. Kitjaroentham, A., Hananantachai, H., Phonrat, B., Preutthipan, S. \& Tungtrongchitr, R. Low density lipoprotein receptor-related protein 5 gene polymorphisms and osteoporosis in Thai menopausal women. J. Negat. Results Biomed. 15, 16 (2016).

11. Canto-Cetina, T. et al. Polymorphism of LRP5, but not of TNFRSF11B, is associated with a decrease in bone mineral density in postmenopausal Maya-Mestizo women. Am. J. Hum. Biol. 25, 713-718 (2013).

12. Funakoshi, Y., Omori, H., Yada, H. \& Katoh, T. A1330V polymorphism of the low-density lipoprotein receptor-related protein 5 gene and bone mineral density in Japanese male workers. Environ. Health Prev. Med. 16, 106-112 (2011).

13. Tran, B. N., Nguyen, N. D., Eisman, J. A. \& Nguyen, T. V. Association between LRP5 polymorphism and bone mineral density: A Bayesian meta-analysis. BMC Med. Genet. 9, 55 (2008).

14. Brixen, K. et al. Polymorphisms in the low-density lipoprotein receptor-related protein 5 (LRP5) gene are associated with peak bone mass in non-sedentary men: Results from the Odense androgen study. Calcif. Tissue Int. 81, 421-429 (2007).

15. Grundberg, E. et al. Large-scale association study between two coding LRP5 gene polymorphisms and bone phenotypes and fractures in men. Osteoporos. Int. 19, 829-837 (2008).

16. Giroux, S., Elfassihi, L., Cardinal, G., Laflamme, N. \& Rousseau, F. LRP5 coding polymorphisms influence the variation of peak bone mass in a normal population of French-Canadian women. Bone 40, 1299-1307 (2007). 
17. Saarinen, A. et al. The A1330V polymorphism of the low-density lipoprotein receptor-related protein 5 gene (LRP5) associates with low peak bone mass in young healthy men. Bone 40, 1006-1012 (2007).

18. Urano, T. \& Inoue, S. Recent genetic discoveries in osteoporosis, sarcopenia and obesity. Endocr. J. 62, 475-484 (2015).

19. Komori, T. Signaling networks in RUNX2-dependent bone development. J. Cell. Biochem. 112, $750-755$ (2011).

20. Baron, R. \& Kneissel, M. WNT signaling in bone homeostasis and disease: From human mutations to treatments. Nat. Med. 19, 179-192 (2013).

21. Han, P., Ivanovski, S., Crawford, R. \& Xiao, Y. Activation of the canonical Wnt signaling pathway induces cementum regeneration. J. Bone Miner. Res. 30, 1160-1174 (2015).

22. Nusse, R. \& Clevers, H. Wnt/beta-catenin signaling, disease, and emerging therapeutic modalities. Cell 169, 985-999 (2017).

23. Staal, F. J., Luis, T. C. \& Tiemessen, M. M. WNT signalling in the immune system: WNT is spreading its wings. Nat. Rev. Immunol. 8, 581-593 (2008).

24. Pongracz, J. E., Parnell, S. M., Jones, T., Anderson, G. \& Jenkinson, E. J. Overexpression of ICAT highlights a role for cateninmediated canonical Wnt signalling in early T cell development. Eur. J. Immunol. 36, 2376-2383 (2006).

25. Chen, B. et al. Small molecule-mediated disruption of Wnt-dependent signaling in tissue regeneration and cancer. Nat. Chem. Biol. 5, 100-107 (2009).

26. da Silva, R. A., Ferreira, P. D., De Rossi, A., Nelson-Filho, P. \& Silva, L. A. Toll-like receptor 2 knockout mice showed increased periapical lesion size and osteoclast number. J. Endod. 38, 803-813 (2012).

27. Goldman, E. S. et al. A mouse model for studying the development of apical periodontitis with Age. Cells 10,671 (2021).

28. Kalatzis-Sousa, N. G., Spin-Neto, R., Wenzel, A., Tanomaru-Filho, M. \& Faria, G. Use of micro-computed tomography for the assessment of periapical lesions in small rodents: A systematic review. Int. Endod. J. 50, 352-366 (2017).

29. Yoneda, N. et al. Development of a root canal treatment model in the rat. Sci. Rep. 7, 3315 (2017).

\section{Acknowledgements}

This study was supported by Grant-in-aid for Scientific Research (19K24143 and 20K09952) from the Japan Society for the Promotion of Science, a Grant from The Nakatomi Foundation.

\section{Author contributions}

H.N., Y.I., T.K., and S.I. conceived the experiments. H.N., Y.I., T.K., S.I., and M.A. performed the experiments. H.N., Y.I., T.K., S.I., and M.A. analyzed the data. H.N., S.I., and M.H. wrote the manuscript. All authors reviewed the manuscript.

\section{Competing interests}

The authors declare no competing interests.

\section{Additional information}

Supplementary Information The online version contains supplementary material available at https://doi.org/ 10.1038/s41598-021-99231-X.

Correspondence and requests for materials should be addressed to S.I.

Reprints and permissions information is available at www.nature.com/reprints.

Publisher's note Springer Nature remains neutral with regard to jurisdictional claims in published maps and institutional affiliations.

Open Access This article is licensed under a Creative Commons Attribution 4.0 International License, which permits use, sharing, adaptation, distribution and reproduction in any medium or format, as long as you give appropriate credit to the original author(s) and the source, provide a link to the Creative Commons licence, and indicate if changes were made. The images or other third party material in this article are included in the article's Creative Commons licence, unless indicated otherwise in a credit line to the material. If material is not included in the article's Creative Commons licence and your intended use is not permitted by statutory regulation or exceeds the permitted use, you will need to obtain permission directly from the copyright holder. To view a copy of this licence, visit http://creativecommons.org/licenses/by/4.0/.

(c) The Author(s) 2021 